\title{
Aortic dilatation in children with chronic kidney disease
}

\author{
Uwe Querfeld $^{1}$ (D) - Dieter Haffner ${ }^{2}$
}

Received: 14 May 2020 / Revised: 15 May 2020 / Accepted: 19 May 2020 / Published online: 29 May 2020

(C) IPNA 2020

\section{Dear Editors,}

Madueme et al. have recently shown that aortic dilatation (AD) may be found in some children with CKD [1]. This may renew interest in a potentially important clinical sign of early vascular remodeling that has been previously described in children and adults with CKD, but may be underdiagnosed. While longitudinal studies in CKD patients to our knowledge are lacking, $\mathrm{AD}$ in $\mathrm{CKD}$ may be regarded as a form of maladaptive repair and may predispose to complications in adulthood which have been linked to CKD: aortic dissection and aneurysm formation.

In their retrospective analysis, Madueme and co-authors reviewed echocardiographic data of 501 patients with mild to moderate $\mathrm{CKD}$ enrolled in the CKiD study and found $\mathrm{AD}$ in 30 children $(6 \%)$. The presence of $\mathrm{AD}$ was associated with high diastolic blood pressure, low body weight, and low body mass index. The presence of protein-energy wasting was the strongest independent predictor of AD. In a previous single center study of patients on dialysis $(n=78)$ or with a kidney transplant $(n=19)$, these authors found a much higher prevalence of $\mathrm{AD}(30.9 \%)$. In a classification and regression tree model, a low body mass index, uncontrolled hypertension, and an increased parathyroid hormone (iPTH) serum level

Uwe Querfeld

uwe.querfeld@charite.de

Dieter Haffner

haffner.dieter@mh-hannover.de

1 Department of Pediatric Gastroenterology, Nephrology and Metabolic Diseases, Charité - Universitätsmedizin Berlin, Augustenburger Platz 1, 13353 Berlin, Germany

2 Department of Paediatric Kidney, Liver and Metabolic Diseases, Hannover Medical School, Carl-Neuberg-Str. 1, D-30625 Hannover, Germany were clinical variables distinguishing subpopulations at high versus low risk for $\mathrm{AD}$ [2].

While both studies of these authors could detect an association of $\mathrm{AD}$ with high blood pressure and low BMI, it is unclear why iPTH levels were not reported in their recent study [1]. Higher iPTH levels could reflect a more advanced mineral bone disorder (MBD), which is closely related to cardiovascular remodeling in CKD, but PTH also has direct vascular effects and is closely associated with protein-energy wasting. We have previously shown the presence of $A D$ in uremic rats with vascular calcification and high iPTH levels and could demonstrate that $\mathrm{AD}$ was prevented by submaximal PTH suppression with either cinacalcet or parathyroidectomy [3]. In view of the broad clinical and experimental evidence implying a role of PTH and CKD-MBD in cardiovascular disease, it would be of interest to get more information on iPTH levels and if available, other markers of CKD/MBD and their associations with $\mathrm{AD}$ in the patients studied by Madueme et al.

\section{References}

1. Madueme PC, Ng DK, Guju L, Longshore L, Moore V, Jefferies L, Warady BA, Furth S, Mitsnefes M (2020) Aortic dilatation in children with mild to moderate chronic kidney disease. Pediatr Nephrol 35:1023-1031

2. Kaddourah A, Uthup S, Madueme P, O'Rourke M, Hooper DK, Taylor MD, Colan SD, Jefferies JL, Rao MB, Goebel J (2015) Prevalence and predictors of aortic dilation as a novel cardiovascular complication in children with end-stage renal disease. Clin Nephrol 83:262-271

3. Jung S, Querfeld U, Muller D, Rudolph B, Peters H, Kramer S (2012) Submaximal suppression of parathyroid hormone ameliorates calcitriol-induced aortic calcification and remodeling and myocardial fibrosis in uremic rats. J Hypertens 30:2182-2191

Publisher's note Springer Nature remains neutral with regard to jurisdictional claims in published maps and institutional affiliations. 